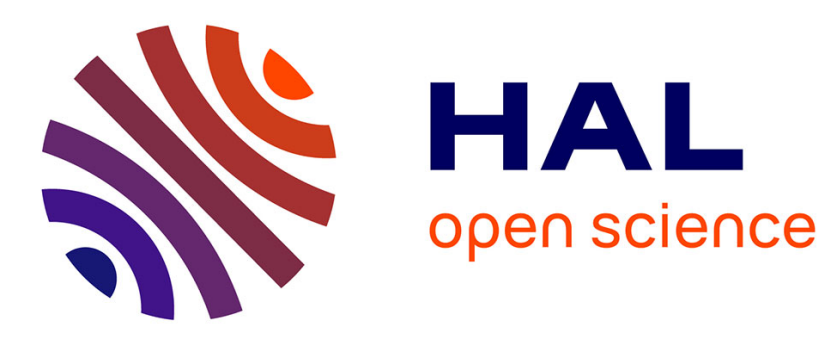

\title{
On the Validation of Homogenized Modeling for Bi-Pantographic Metamaterials via Digital Image Correlation
}

\author{
Emilio Barchiesi, Francesco Dell'Isola, François Hild
}

\section{- To cite this version:}

Emilio Barchiesi, Francesco Dell'Isola, François Hild. On the Validation of Homogenized Modeling for Bi-Pantographic Metamaterials via Digital Image Correlation. International Journal of Solids and Structures, 2021, 208-209, pp.49-62. 10.1016/j.ijsolstr.2020.09.036 . hal-02954418

\author{
HAL Id: hal-02954418 \\ https://hal.science/hal-02954418
}

Submitted on 1 Oct 2020

HAL is a multi-disciplinary open access archive for the deposit and dissemination of scientific research documents, whether they are published or not. The documents may come from teaching and research institutions in France or abroad, or from public or private research centers.
L'archive ouverte pluridisciplinaire HAL, est destinée au dépôt et à la diffusion de documents scientifiques de niveau recherche, publiés ou non, émanant des établissements d'enseignement et de recherche français ou étrangers, des laboratoires publics ou privés. 


\title{
On the Validation of Homogenized Modeling for Bi-Pantographic Metamaterials via Digital Image Correlation
}

\author{
Emilio Barchiesi ${ }^{\mathrm{a}, *}$, Francesco dell'Isola $^{\mathrm{c}}$, François Hild ${ }^{\mathrm{b}}$ \\ ${ }^{a}$ Dipartimento di Ingegneria Strutturale e Geotecnica \\ Università degli Studi di Roma "La Sapienza", Italy \\ International Research Center M\&MoCS \\ Università degli Studi dell'Aquila, Italy \\ ${ }^{b}$ Université Paris-Saclay, ENS Paris-Saclay, CNRS \\ LMT-Laboratoire de Mécanique et Technologie, Gif-sur-Yvette, France \\ ${ }^{c}$ Dipartimento di Ingegneria Civile, Edile-Architettura e Ambientale \\ International Research Center M\&MoCS \\ Università degli Studi dell'Aquila, Italy
}

\begin{abstract}
The derivation by variational asymptotic homogenization of a 2D-continuum model describing large elastic planar deformations of a discrete bi-pantographic structure is presented. A rectangular bi-pantographic specimen was additively manufactured and subjected to a bias extension test for macroscopic strains up to ca. $40 \%$. The deformations of the bi-pantographic sample were measured via FE-based digital image correlation. Measured boundary conditions then drove the numerical model of the experiment. The gray level residuals were utilized to independently probe the kinematic hypotheses of DIC and FE simulations against the full video of the experiment for validation purposes.

Keywords: Metamaterial, second gradient continuum, Hencky-type model, additive manufacturing, bias extension test
\end{abstract}

\footnotetext{
${ }^{*}$ Corresponding author

Email address: BarchiesiEmilio@gmail.com (Emilio Barchiesi)
} 


\section{Introduction}

In the last decade, motivated by the application of generalized and second gradient continua theories introduced in the early 1960s [1, 2, 3, 4], mechanical models of pantographic structures [5, 6] have been investigated. Owing to 5 their simple topology, pantographic fabrics are an archetype when dealing with the homogenization of discrete structures leading to second gradient continua [7, 8], discrete [9, 10, 11, 12, 13, 14, and semi-discrete [15] models making use of extension and bending elements [16, 17, 18, 19], generalized shell theories [20, 21, 22], second gradient continuaand related mathematical challenges [23, 24], parameter identification of second gradient continua [25, 26], numerical simulations dealing with second gradient continua [27, 28, 29, 30, continuum descriptions of fibrous materials [31, among other fields in Solid Mechanics.

Interestingly, the scientific developments of pantographic fabrics can be also 15 recast within the spirit of metamaterials [32, 33]. Pantographic prototypes have been developed and manufactured with the goal of obeying some of the above mentioned models and, consequently, material control by optimization of additive manufacturing processes at relevant scales has been considered a key objective. Therefore, experimental studies have been carried out for understanding how different 3D-printing processes and raw materials 34 influenced mechanical and morphological properties of printed samples at different scales, ranging from micrometers [35] to millimeters, which were imaged by optical and Scanning Electron microscopy, and tomography.

Studies analyzing different designs (e.g., hinge/torsional joints [36]) have also been carried out. Bridging properties at different scales [37, 38] has led to new methodologies for, say, granular microstructures [39, 40, 41]. Experimental studies have shown that pantographic fabrics exhibited a remarkably wide elastic domain and that the elastic response of printed specimens depended weakly upon the raw printed material [42, thereby suggesting that they can be considered by all means as metamaterials [43. Such studies have 
also unveiled new phenomenologies, for instance, the so-called Poynting effect reversal [44, which has then led to new model features. It has been proven that damage mechanisms strongly depended upon geometric dimensions of mechanical elements and raw material properties [45]. In all analyzed cases

35 of printed specimens, thanks to their reticulated structure, they were shown to be damage tolerant [9].

The use of digital image correlation (DIC) as a means of analyzing kinematic details in experiments performed on such materials has been considered very recently. Finite element based analyses have been performed at macroscopic [46]

40 and mesoscopic [47 scales. In the latter case, the underlying mesh was directly adapted by using basic morphological operations applied to the picture of the reference configuration. An alternative approach consisted in starting with the mesh of the nominal configuration, and use regularized registrations to backtrack it onto the actual picture of the reference configuration [48. This measurement

45 technique is very appealing since these metamaterials deform considerably and classical means (e.g., strain gauges or extensometer) cannot be used.

The development of bi-pantographic fabrics has benefitted from the abovementioned studies. Such metamaterials were initially proposed as assemblies of discrete pantographic beams [49] leading, at the macroscopic scale, to second so gradient materials whose strain energy density depends upon the curvature and the stretch derivative of material lines along the corresponding reference direction. This first work was restricted to small strains and non-extensible elements. It is proposed to derive hereafter, by asymptotic homogenization, the macroscopic response of a $2 \mathrm{D}$-continuum, which is able to describe large strains of bi-pantographic metamaterials.

In the present study, a homogenized model of bi-pantographic fabrics was also probed against experimental measurements provided by global (i.e., FEbased) DIC. Consequently, the finite element discretizations can be made identical in DIC analyses and numerical simulations. Figure 1 shows the

6o flowchart of the one-way validation framework followed herein. For a given picture of the deformed configuration, the displacement field is computed as the 
result of the registration with the picture in the reference configuration. The quality of the registration is assessed thanks to the gray level residuals. The measured Dirichlet boundary conditions are then applied to the finite element model. For the same picture, the displacement field is computed according to the implemented constitutive law. Because the measured boundary conditions were applied to the FE model, gray level residuals can also be evaluated [50]. It was shown that such procedure was the best when probing multiscale models [51]. Thanks to such procedure, the quality of the measurements and the predictions is independently probed with respect to the experimental data (i.e., the full video acquired during the test).

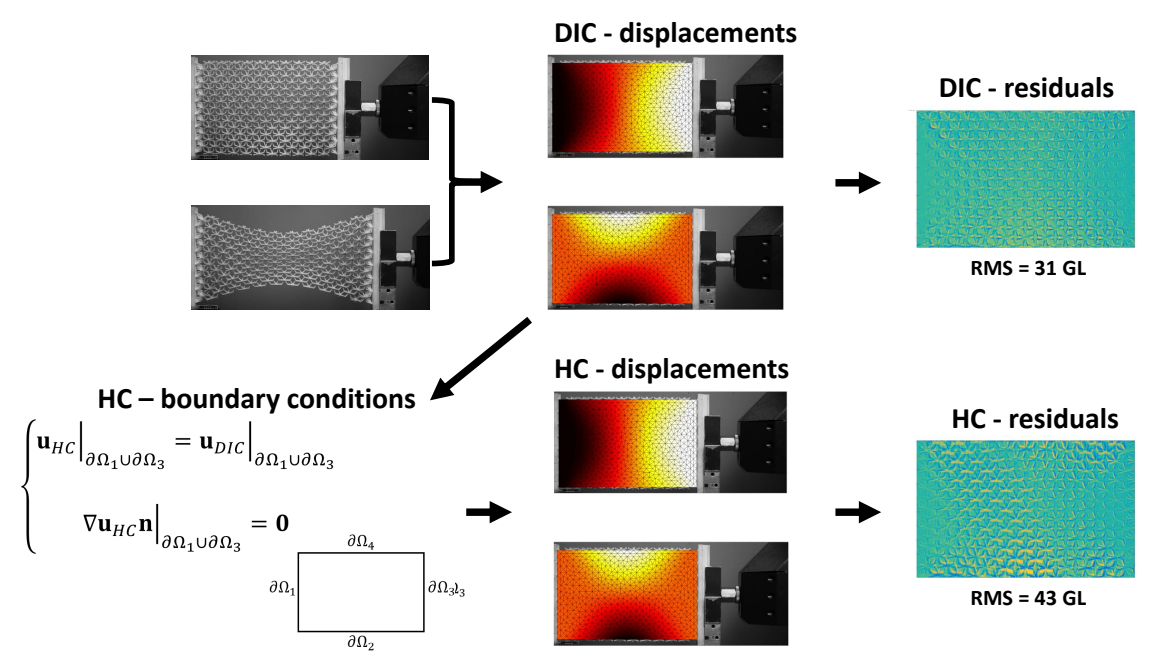

Figure 1: Flowchart of the validation procedure comparing DIC and homogenized continuum residuals in a bias extension test.

It has to be noted that the methodologies exploited in the global T3-DIC analysis, with a mesh made of 3-noded (T3) triangles that was not made to match the bi-pantographic unit cells, are not new [46. However, the oneway connection between experimental measurements and simulations is here established for the first time in the literature for the quantitative validation of a homogenized model of bi-pantographic fabrics. 
Lower-scale, i.e., local mechanical and DIC analyses considering richer kinematic hypotheses and geometries could achieve better results in terms

so of gray level residuals [52. Yet, the question that is addressed herein is to assess to what extent a coarse macroscale continuous description can represent and predict experimental measurements. Such reduced-order description is of utmost utility when dealing with complex systems whose efficient element-byelement description still poses challenges to modern computational methods.

85 The problem of finding an efficient model for the studied metamaterial is the scope of the present paper. For a consistent comparison between reducedorder model and DIC, both will employ the same (macroscale) geometry and kinematics. This goal motivates the use of FE-based DIC [53, 54, 55], where the underlying hypothesis is the continuity of the displacement field associated

so with FE discretizations. Last, it is noteworthy that such macroscale analyses would clearly compare better with experimental pictures should the number of unit cells be increased, as the scale separation would become more pronounced.

The outline of the paper follows the previous flowchart. In Section 2 the main steps of the homogenization procedures are presented. The boundary 95 value problem is introduced for the considered bias extension test. The latter is studied in Section 3, and global DIC is briefly recalled. A mesh sensitivity analysis is performed to validate the chosen discretization for DIC measurements. Section 4 is devoted to the validation of the derived model against experimental data extracted from a bias extension test.

\section{Homogenized Continuum (HC)}

\subsection{Studied Metamaterial}

Following the rationale behind the design of pantographic prototypes [49], a rectangular bi-pantographic specimen was additively manufactured by Selective Laser Sintering (SLS) using Polyamide as the bulk material (Figure 2). 


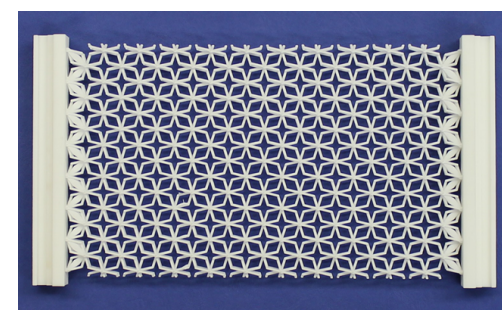

(a)

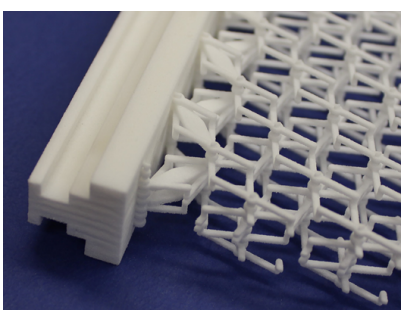

(b)

Figure 2: Additively manufactured bi-pantographic specimen. Full top-view (a) and zoomed in left-bottom area (b) showing ends to be put in grips, element arrangement and connections.

In the reference configuration (Figure 3(a)), the discrete bi-pantographic structure is formed by square cells (see Figure $3(\mathrm{~b})$ ) whose sides have a length $\sqrt{2} \varepsilon=17 \mathrm{~mm}$, arranged along straight lines within the reference rectangular domain $\Omega$, in the direction of the unit basis vectors $\mathbf{e}_{\zeta}, \mathbf{e}_{\varsigma} \in \mathbb{E}^{2}$. The edges of $\Omega(L=187 \mathrm{~mm}$ and $\ell=119 \mathrm{~mm})$ constituting subsets of its boundary $\partial \Omega$ are denoted as $\partial \Omega_{k}, k \in\{1 \ldots 4\}$.

a)

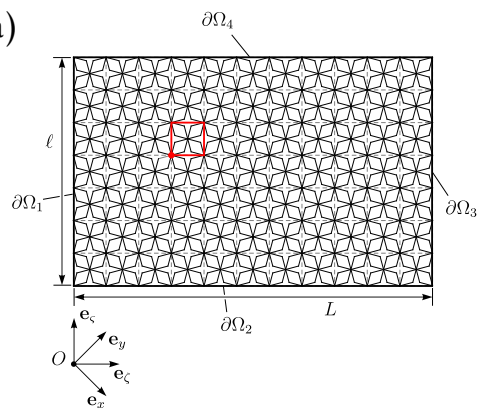

b)

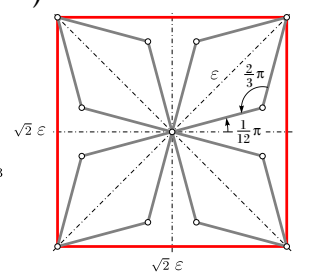

c)

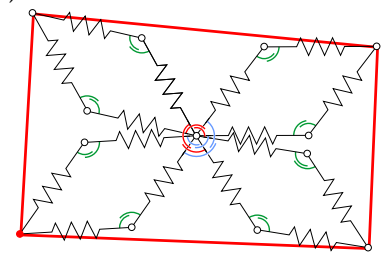

Figure 3: Bi-pantographic metamaterial. (a) Domain $\Omega$. (b) Reference configuration of a unit cell. (c) Force elements and deformed configuration of a unit cell.

\subsection{Homogenization Scheme}

In Figure 3.c), elastic elements are colored in black (extensional Hooke elastic springs, stiffness $k_{E}$ ), red (rotational Hooke elastic springs, stiffness $k_{F}$ ), blue (rotational Hooke elastic springs, stiffness $k_{F}$ ) and green (rotational 115 Hooke elastic springs, stiffness $k_{S}$ ). It is worth emphasizing at this stage 
that the passage from pantographic [6] to bi-pantographic fabrics, in terms of asymptotic homogenization, is not as trivial as the designation may suggest. Bi-pantographic fabrics are regarded as assemblies of two orthogonal families of parallel $\varepsilon$-spaced pantographic beams (Figure $4(\mathrm{a})$ ), hinge-joined together at their intersection points, and aligned along $\mathbf{e}_{x}$ and $\mathbf{e}_{y}$, respectively. First, the homogenization of a single pantographic beam is addressed [56].

a)

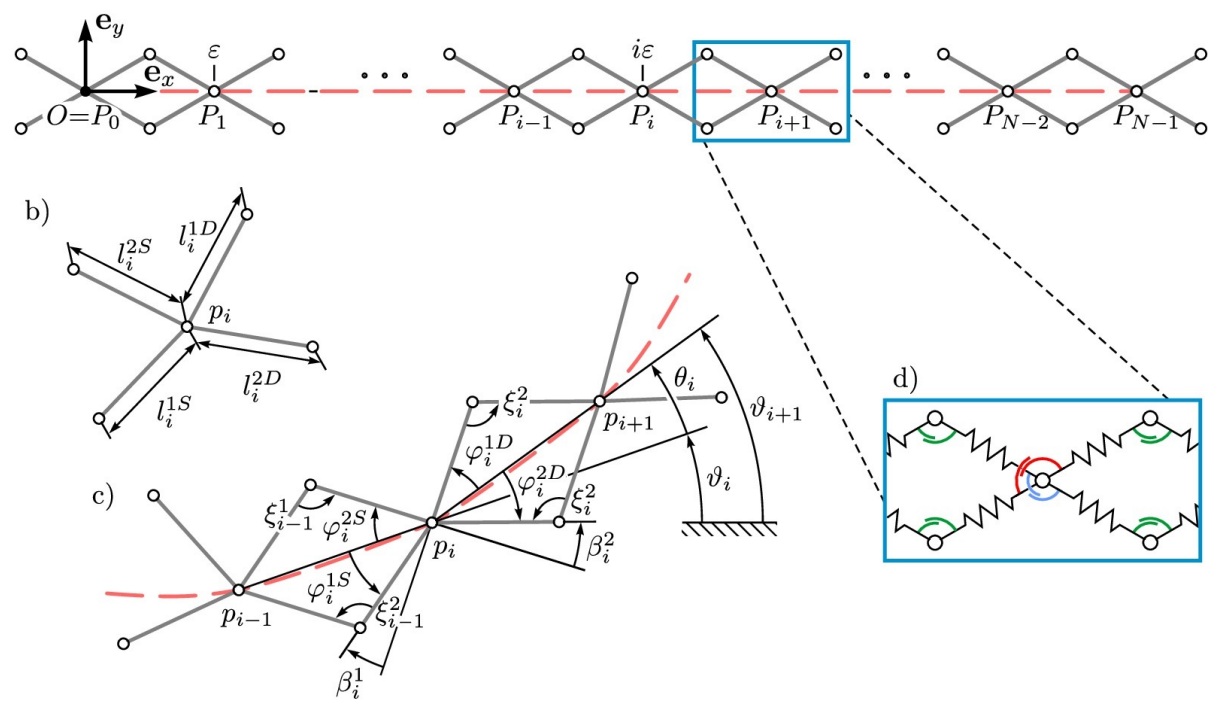

Figure 4: Schematic view of a discrete pantographic beam. (a) Reference configuration. (b) Generalized coordinates of $i$-th cell. (c) Deformed configuration with redundant kinematic quantities. (d) Force elements of a single cell.

When not otherwise specified, the indices $i, \mu$ and $\nu$ will henceforth belong respectively to the following index sets: $i \in\{0,1, \ldots, N-1\}, \mu \in\{1,2\}$ and $\nu \in\{D, S\}$. Throughout the homogenization procedure, it is assumed that the angles $\varphi_{i}^{1 D}$ and $\varphi_{i}^{2 D}$ vary within the range $(0, \pi)$, thus entailing that $\xi_{i}^{\mu} \in(0, \pi)$. In such a case, the kinematics of the spring system of Figure 4 is described by finitely many generalized coordinates. The coordinates are the positions $\mathbf{p}_{i} \in \mathbb{E}^{2}$ of the points at position $P_{i}$ in the reference configuration and the lengths of the 
oblique deformed springs $\ell_{i}^{\mu \nu} \in \mathbb{R}$. The angles $\varphi_{i}^{\mu \nu}$ in Figure 4(c) are obtained by applying the law of cosines

$$
\begin{aligned}
& \varphi_{i}^{1 D}=\cos ^{-1}\left[\frac{\left\|\mathbf{p}_{i+1}-\mathbf{p}_{i}\right\|^{2}+\left(\ell_{i}^{1 D}\right)^{2}-\left(\ell_{i+1}^{2 S}\right)^{2}}{2 \ell_{i}^{D}\left\|\mathbf{p}_{i+1}-\mathbf{p}_{i}\right\|}\right], \\
& \varphi_{i}^{1 S}=\cos ^{-1}\left[\frac{\left\|\mathbf{p}_{i}-\mathbf{p}_{i-1}\right\|^{2}+\left(\ell_{i}^{1 S}\right)^{2}-\left(\ell_{i-1}^{2 D}\right)^{2}}{2 \ell_{i}^{1 S}\left\|\mathbf{p}_{i}-\mathbf{p}_{i-1}\right\|}\right], \\
& \varphi_{i}^{2 D}=\cos ^{-1}\left[\frac{\left\|\mathbf{p}_{i+1}-\mathbf{p}_{i}\right\|^{2}+\left(\ell_{i}^{2 D}\right)^{2}-\left(\ell_{i+1}^{1 S}\right)^{2}}{2 \ell_{i}^{2 D}\left\|\mathbf{p}_{i+1}-\mathbf{p}_{i}\right\|}\right], \\
& \varphi_{i}^{2 S}=\cos ^{-1}\left[\frac{\left\|\mathbf{p}_{i}-\mathbf{p}_{i-1}\right\|^{2}+\left(\ell_{i}^{2 S}\right)^{2}-\left(\ell_{i-1}^{1 D}\right)^{2}}{2 \ell_{i}^{2 S}\left\|\mathbf{p}_{i}-\mathbf{p}_{i-1}\right\|}\right]
\end{aligned}
$$

while the angles $\xi_{i}^{\mu}$ in Figure 4 (c) are computed as

$$
\xi_{i}^{1(2)}=\cos ^{-1}\left[\frac{\left(\ell_{i}^{1(2) D}\right)^{2}+\left(\ell_{i+1}^{2(1) S}\right)^{2}-\left\|\mathbf{p}_{i+1}-\mathbf{p}_{i}\right\|^{2}}{2 \ell_{i}^{1(2) D} \ell_{i+1}^{2(1) S}}\right] .
$$

The angle $\theta_{i}$ in Figure $4(\mathrm{c})$ reads

$$
\theta_{i}=\vartheta_{i+1}-\vartheta_{i}=\tan ^{-1}\left[\frac{\left(\mathbf{p}_{i+1}-\mathbf{p}_{i}\right) \cdot \mathbf{e}_{y}}{\left(\mathbf{p}_{i+1}-\mathbf{p}_{i}\right) \cdot \mathbf{e}_{x}}\right]-\tan ^{-1}\left[\frac{\left(\mathbf{p}_{i}-\mathbf{p}_{i-1}\right) \cdot \mathbf{e}_{y}}{\left(\mathbf{p}_{i}-\mathbf{p}_{i-1}\right) \cdot \mathbf{e}_{x}}\right] .
$$

The deformation energy of the discrete micromodel is expressed as

$$
\begin{aligned}
\mathcal{E}^{\mu} & =\frac{k_{E}}{2} \sum_{i} \sum_{\mu, \nu}\left(\ell_{i}^{\mu \nu}-\frac{1}{\sqrt{3}} \varepsilon\right)^{2}+\frac{k_{F}}{2} \sum_{i} \sum_{\mu}\left(\beta_{i}^{\mu}\right)^{2}+\frac{k_{S}}{2} \sum_{i} \sum_{\mu}\left(\xi_{i}^{\mu}-\pi+2 \gamma\right)^{2} \\
& =\frac{k_{E}}{2} \sum_{i} \sum_{\mu, \nu}\left(\ell_{i}^{\mu \nu}-\frac{1}{\sqrt{3}} \varepsilon\right)^{2}+\frac{k_{F}}{2} \sum_{i} \sum_{\mu}\left[\theta_{i}+(-1)^{\mu}\left(\varphi_{i}^{\mu S}-\varphi_{i}^{\mu D}\right)\right]^{2} \\
& +\frac{k_{S}}{2} \sum_{i=0}^{N-2} \sum_{\mu}\left(\xi_{i}^{\mu}-\frac{2}{3} \pi\right)^{2}
\end{aligned}
$$

where $k_{E}>0$ and $k_{F}, k_{S}>0$ are the stiffnesses of the extensional and rotational springs, respectively.

The following asymptotic expansion is assumed for the lengths $\ell_{i}^{\mu \nu}$ of the extensional springs

$$
\ell_{i}^{\mu \nu}=\frac{1}{\sqrt{3}} \varepsilon+\varepsilon^{2} \tilde{\ell}_{i}^{\mu \nu}+o\left(\varepsilon^{2}\right), \quad \tilde{\ell}_{i}^{\mu \nu} \in \mathbb{R} .
$$


Assumption (5) states that the lengths $\ell_{i}^{\mu \nu}$ are obtained as $\varepsilon$-corrections of the initial length $\frac{1}{\sqrt{3}} \varepsilon$. Therefore, the lengths $\ell_{i}^{\mu \nu}$ are vanishing with order one in $\varepsilon$ when $\varepsilon \rightarrow 0$. In other words, a kind of quasi-inextensibility property is assumed for the extensional springs, whose elongation is given, at leading order in $\varepsilon$, by $\varepsilon^{2} \tilde{\ell}_{i}^{\mu \nu}$. Therefore, the quantities $\tilde{\ell}_{i}^{\mu \nu}$, independent of $\varepsilon$, are $\varepsilon^{2}$-scaled (absolute) elongations of extensional springs. Inserting assumption (5) into the energy (4) leads to

$$
\begin{aligned}
\mathcal{E}^{\mu} & =\frac{k_{E}}{2} \sum_{i} \sum_{\mu, \nu}\left[\varepsilon^{2} \tilde{\ell}_{i}^{\mu \nu}+o\left(\varepsilon^{2}\right)\right]^{2} \\
& +\frac{k_{F}}{2} \sum_{i} \sum_{\mu}\left[\theta_{i}+(-1)^{\mu}\left(\varphi_{i}^{\mu S}-\varphi_{i}^{\mu D}\right)\right]^{2}+\frac{k_{S}}{2} \sum_{i}^{N-2} \sum_{\mu}\left(\xi_{i}^{\mu}-\frac{2}{3} \pi\right)^{2} .
\end{aligned}
$$

Due to the slenderness of the microstructure, a macroscale one-dimensional continuum is sought in the limit of vanishing $\varepsilon$. The reference domain of the continuum is a one-dimensional straight segment connecting all points $P_{i}$ of the discrete micromodel. An abscissa $s$ is introduced and varies within the interval $I=[0,(N-1) \varepsilon]$. The independent kinematic Lagrangian descriptors of the macromodel are assumed to be $\chi: I \rightarrow \mathbb{E}^{2}$ and $\tilde{\ell}^{\mu \nu}: I \rightarrow \mathbb{R}$. The placement field $\chi$ locates the $1 \mathrm{D}$-continuum into $\mathbb{E}^{2}$, and is introduced to describe, at the macroscale, the midline of the discrete system (i.e., points $\mathbf{p}_{i} \in \mathbb{E}^{2}$ ). Accounting for the change in spring lengths $\tilde{\ell}_{i}^{\mu \nu}$ introduced in Equation (5), the placement field is augmented by four micro-strain functions $\tilde{\ell}^{\mu \nu}$. The generalized coordinates of the discrete system are related to $\chi$ and $\tilde{\ell}^{\mu \nu}$ by

$$
\chi\left(s_{i}\right)=\mathbf{p}_{i}, \quad \tilde{\ell}^{\mu \nu}\left(s_{i}\right)=\tilde{\ell}_{i}^{\mu \nu}
$$

where $s_{i}=i \varepsilon$. For convenience, the functions $\rho:[0, L] \rightarrow \mathbb{R}^{+}$and $\vartheta: I \rightarrow$ $[0,2 \pi)$ are introduced to rewrite the tangent vector field $\chi^{\prime}$ to the deformed $1 \mathrm{D}$-continuum as

$$
\chi^{\prime}(s)=\rho(s)\left[\cos \vartheta(s) \mathbf{e}_{x}+\sin \vartheta(s) \mathbf{e}_{y}\right]
$$

where $(\cdot)^{\prime}$ denotes the differentiation of $(\cdot)$ with respect to the reference abscissa $s$. Thus $\rho$ corresponds to the norm of the tangent vector $\left\|\chi^{\prime}\right\|$, and is referred to 
as stretch of the 1D-continuum and $\vartheta^{\prime}$ corresponds to the material curvature of such continuum. For the asymptotic identification, the energy (6) is expanded in $\varepsilon$. First, the expansion of $\chi$ is given by

$$
\chi\left(s_{i \pm 1}\right)=\chi\left(s_{i}\right) \pm \varepsilon \chi^{\prime}\left(s_{i}\right)+\frac{\varepsilon^{2}}{2} \chi^{\prime \prime}\left(s_{i}\right)+o\left(\varepsilon^{2}\right),
$$

while for $\tilde{\ell}^{\mu \nu}$, it becomes

$$
\ell^{\mu \nu}\left(s_{i \pm 1}\right)=\frac{1}{\sqrt{3}} \varepsilon+\tilde{\ell}^{\mu \nu}\left(s_{i}\right) \varepsilon^{2}+o\left(\varepsilon^{2}\right)
$$
expansion $\tilde{\ell}^{\mu \nu}\left(s_{i \pm 1}\right)=\tilde{\ell}^{\mu \nu}\left(s_{i}\right)+o\left(\varepsilon^{0}\right)$.

Next, the aim is to expand Equation (6). The terms $\theta_{i}, \varphi_{i}^{\mu S}-\varphi_{i}^{\mu D}$ and $\xi_{i}^{\mu}$, which are all functions of the placement field $\chi$ and/or the micro-strains $\tilde{\ell}^{\mu \nu}$, need to be approximated. Combining Equations (9) and (7) $)_{1}$ with Equation (3) 170 yields

$$
\theta_{i}=\vartheta^{\prime}\left(s_{i}\right) \varepsilon+o(\varepsilon)
$$

Similarly, combining Equations 9 (10) and (7) with Equation (1) leads to

$$
\begin{aligned}
& \varphi_{i}^{1(2) S}-\varphi_{i}^{1(2) D}= \\
& \left.\frac{4\left[\rho^{2}-2 / 3\right]\left(\tilde{\ell}^{1(2) S}-\tilde{\ell}^{1(2) D}\right)+(2 / \sqrt{3})\left(\rho^{2}\right)^{\prime}+(8 / 3)\left(\tilde{\ell}^{2(1) D}-\tilde{\ell}^{2(1) S}\right)}{\rho(4 / \sqrt{3}) \sqrt{4 / 3-\rho^{2}}}\right|_{s=s_{i}} ^{\varepsilon+o(\varepsilon)}
\end{aligned}
$$

for the differences $\varphi_{i}^{1(2) S}-\varphi_{i}^{1(2) D}$. By incorporating Equations (9), 10, and (7) in Equation (2) enables the angles $\xi_{i}^{\mu}$ to be assessed

$$
\xi_{i}^{\mu}=\left.\cos ^{-1}\left(1-\frac{3}{2} \rho^{2}\right)\right|_{s=s_{i}}+o\left(\varepsilon^{0}\right) .
$$

Substituting Equations (11), 12 and (13) in Equation (6) provides the 175 expansion of the micromodel energy as a function of the kinematic descriptors 
$\chi$ and $\tilde{\ell}^{\mu \nu}$

$$
\begin{aligned}
\mathcal{E}^{\mu} & =\sum_{i}\left\{\frac{k_{E} \varepsilon^{4}}{2}\left[\sum_{\mu, \nu}\left(\tilde{\ell}^{\mu \nu}\right)^{2}+o\left(\varepsilon^{0}\right)\right]+k_{S}\left[\cos ^{-1}\left(1-\frac{3}{2} \rho^{2}\right)-\frac{2}{3} \pi+o\left(\varepsilon^{0}\right)\right]^{2}\right. \\
& +\frac{k_{F} \varepsilon^{2}}{2}\left[\vartheta^{\prime}+\frac{\left.4\left[\rho^{2}-2 / 3\right)\right]\left(\tilde{\ell}^{1 S}-\tilde{\ell}^{1 D}\right)+(2 / \sqrt{3})\left(\rho^{2}\right)^{\prime}+(8 / 3)\left(\tilde{\ell}^{2 D}-\tilde{\ell}^{2 S}\right)}{4 \rho(1 / \sqrt{3}) \sqrt{4 / 3-\rho^{2}}}+o\left(\varepsilon^{0}\right)\right]^{2} \\
& \left.+\frac{k_{F} \varepsilon^{2}}{2}\left[\vartheta^{\prime}+\frac{\left.4\left[\rho^{2}-2 / 3\right)\right]\left(\tilde{\ell}^{2 S}-\tilde{\ell}^{2 D}\right)+(2 / \sqrt{3})\left(\rho^{2}\right)^{\prime}+(8 / 3)\left(\tilde{\ell}^{1 D}-\tilde{\ell}^{1 S}\right)}{4 \rho(1 / \sqrt{3}) \sqrt{4 / 3-\rho^{2}}}+o\left(\varepsilon^{0}\right)\right]^{2}\right\}_{s=s_{i}} .
\end{aligned}
$$

The stiffnesses of the discrete system are related to the microscopic length scale $\varepsilon$ by the following scaling laws

$$
k_{E}=K_{E} \varepsilon^{-3}, \quad k_{F}=K_{F} \varepsilon^{-1}, \quad k_{S}=K_{S} \varepsilon
$$

where $K_{E}, K_{F}, K_{S}>0$ are constants that are independent of $\varepsilon$. It is worth noting that the asymptotic expansion (5) is implied by the scaling laws (15), which are such that $k_{F} / k_{E} \propto \varepsilon^{2}$ and $k_{S} / k_{E} \propto \varepsilon^{4}$, meaning that extensional springs are becoming stiffer than rotational springs (with different orders in $\varepsilon$ according to the type of spring $(\mathrm{F} / \mathrm{S}))$ as $\varepsilon$ tends to zero.

The continuum limit is now obtained by letting $\varepsilon \rightarrow 0$. Using Equation (14) together with the scaling laws (15) allows the deformation energy to be derived for the homogenized model

$$
\begin{aligned}
\mathcal{E} & =\int_{I}\left\{K_{S}\left[\cos ^{-1}\left(1-\frac{3}{2} \rho^{2}\right)-\frac{2}{3} \pi\right]^{2}+\frac{K_{E}}{2} \sum_{\mu \nu}\left(\tilde{\ell}^{\mu \nu}\right)^{2}\right\} \mathrm{d} s \\
& +\int_{I} \frac{K_{F}}{2}\left[\vartheta^{\prime}+\frac{\left.4\left[\rho^{2}-2 / 3\right)\right]\left(\tilde{\ell}^{1 S}-\tilde{\ell}^{1 D}\right)+(2 / \sqrt{3})\left(\rho^{2}\right)^{\prime}+(8 / 3)\left(\tilde{\ell}^{2 D}-\tilde{\ell}^{2 S}\right)}{4 \rho(1 / \sqrt{3}) \sqrt{4 / 3-\rho^{2}}}\right]^{2} \mathrm{~d} s \\
& +\int_{I} \frac{K_{F}}{2}\left[\vartheta^{\prime}+\frac{\left.4\left[\rho^{2}-2 / 3\right)\right]\left(\tilde{\ell}^{2 S}-\tilde{\ell}^{2 D}\right)+(2 / \sqrt{3})\left(\rho^{2}\right)^{\prime}+(8 / 3)\left(\tilde{\ell}^{1 D}-\tilde{\ell}^{1 S}\right)}{4 \rho(1 / \sqrt{3}) \sqrt{4 / 3-\rho^{2}}}\right]^{2} \mathrm{~d} s .
\end{aligned}
$$

The energy is enforced to be stationary with respect to the independent kinematic descriptors $\tilde{\ell}^{\mu \nu}$. Equating to zero the variations of the deformation energy functional (16) with respect to admissible variations in the independent kinematic descriptors $\tilde{\ell}^{\mu \nu}$ yields a linear system of four algebraic equations in 
which $\tilde{\ell}^{\mu \nu}$ are the unknowns. Introducing the quantities

$$
C_{1}=\frac{K_{F}}{2 K_{F} \rho^{2}-1 / 3\left(K_{E} \rho^{2}+8 K_{F}\right)}, \quad C_{2}=\frac{K_{F} \sqrt{4 / 3-\rho^{2}}}{(1 / 3) K_{E} \rho^{2}-2 K_{F} \rho^{2}-(4 / 9) K_{E}},
$$

at equilibrium, the following conditions are satisfied

$$
\tilde{\ell}^{\mu D}=\frac{1}{\sqrt{3}} \rho\left[\rho^{\prime} C_{1}+(-1)^{\mu-1} \vartheta^{\prime} C_{2}\right], \quad \tilde{\ell}^{\mu S}=\frac{1}{\sqrt{3}} \rho\left[-\rho^{\prime} C_{1}+(-1)^{\mu} \vartheta^{\prime} C_{2}\right] .
$$

By substituting the results (18) into Equation (16), the homogenized deformation energy is expressed in terms of the placement field $\chi$ only

$$
\begin{aligned}
\mathcal{E} & =\int_{I}\left\{K _ { E } K _ { F } \left[\frac{3 / 4 \rho^{2}-1}{3 / 4 \rho^{2}\left(K_{E}-6 K_{F}\right)-K_{E}} \vartheta^{\prime 2}\right.\right. \\
& \left.+\frac{3 / 4 \rho^{2}}{\left(1-3 / 4 \rho^{2}\right)\left[8 K_{F}+\rho^{2}\left(K_{E}-6 K_{F}\right)\right]} \rho^{\prime 2}\right] \\
& \left.+K_{S}\left[\cos ^{-1}\left(1-\frac{3}{2} \rho^{2}\right)-\frac{2}{3} \pi\right]^{2}\right\} \mathrm{d} s .
\end{aligned}
$$

The results obtained thus far for a single pantographic beam are extended to homogenize the behavior of bi-pantographic metamaterials. Let $\Psi\left(\rho, \rho^{\prime}, \vartheta^{\prime}\right)$ denote the integrand of Equation $(19)$. The domain $\Omega$ is expressed as $\Omega=$ $\left\{\mathbf{x}=(x, y) \in \mathbb{R}^{2}\right.$ s.t. $\left.x \in \tilde{X} \wedge y \in \tilde{Y}(x)\right\}=\left\{\mathbf{x}=(x, y) \in \mathbb{R}^{2}\right.$ s.t. $y \in$ $\bar{Y} \wedge x \in \bar{X}(y)\}$, where $(x, y)$ are the coordinates of $\mathbf{x}$ in the basis $\left(\mathbf{e}_{x}, \mathbf{e}_{y}\right)$. The family of pantographic beams within $\Omega$ aligned along $\mathbf{e}_{x}$ in the reference configuration is now considered. The corresponding placement field $\chi_{x}: \Omega \rightarrow$ $\mathbb{E}^{2}$ is defined to be such that $\chi_{x}(\mathbf{x})$ denotes the current position of the hinge point of a beam belonging to such a family, which is at position $\mathbf{x}$ in the reference configuration. Similarly, the placement field $\chi_{y}: \Omega \rightarrow \mathbb{E}^{2}$ is defined for the family of pantographic beams aligned along $\mathbf{e}_{y}$.

The hinge constraint applied to the intersection $\mathbf{x}$ (reference configuration) of the two families of pantographic beams is encoded in expressions $\chi_{x}(\mathbf{x})=$ $\chi_{y}(\mathbf{x})=\chi_{H C}(\mathbf{x}):=\mathbf{u}_{H C}(\mathbf{x})-\mathbf{x}$, where $\chi_{H C}: \Omega \rightarrow \mathbb{E}^{2}$ and $\boldsymbol{u}_{H C}: \Omega \rightarrow \mathbb{R}^{2}$ are the placement and displacement functions of the target homogenized continuum, 210 respectively. The deformation energy of the bi-pantographic structure is given as the sum of the deformation energies of the two families of pantographic 
beams. Hence, the homogenized deformation energy for the bi-pantographic metamaterial is expressed as

$$
\begin{aligned}
\mathcal{E} & =\int_{\tilde{X}} \int_{\tilde{Y}(x)} \Psi\left(\rho_{y}(\mathbf{x}), \frac{\partial \rho_{y}}{\partial y}(\mathbf{x}), \frac{\partial \vartheta_{y}}{\partial y}(\mathbf{x})\right) \mathrm{d} y \mathrm{~d} x \\
& +\int_{\bar{Y}} \int_{\bar{X}(y)} \Psi\left(\rho_{x}(\mathbf{x}), \frac{\partial \rho_{x}}{\partial x}(\mathbf{x}), \frac{\partial \vartheta_{x}}{\partial x}(\mathbf{x})\right) \mathrm{d} x \mathrm{~d} y,
\end{aligned}
$$

where $\rho_{x}, \rho_{y}: \Omega \rightarrow \mathbb{R}^{+}$and $\vartheta_{x}, \vartheta_{y}: \Omega \rightarrow[0,2 \pi)$ are implicitly defined by

$$
\begin{aligned}
& \frac{\partial \boldsymbol{\chi}_{H C}}{\partial x}(\mathbf{x})=\rho_{x}(\mathbf{x})\left\{\left[\cos \vartheta_{x}(\mathbf{x})\right] \mathbf{e}_{x}+\left[\sin \vartheta_{x}(\mathbf{x})\right] \mathbf{e}_{y}\right\} \\
& \frac{\partial \boldsymbol{\chi}_{H C}}{\partial y}(\mathbf{x})=\rho_{y}(\mathbf{x})\left\{\left[\cos \vartheta_{y}(\mathbf{x})\right] \mathbf{e}_{y}+\left[\sin \vartheta_{y}(\mathbf{x})\right] \mathbf{e}_{x}\right\}
\end{aligned}
$$

215

and, with the previous derivation, reduce to

$$
\begin{aligned}
\mathcal{E} & =\int_{\Omega} \sum_{\alpha=\{x, y\}}\left\{K_{S}\left[\cos ^{-1}\left(1-\frac{3}{2} \rho_{\alpha}^{2}\right)-\frac{2}{3} \pi\right]^{2}\right. \\
& +K_{E} K_{F}\left[\frac{3 / 4 \rho_{\alpha}^{2}-1}{3 / 4 \rho_{\alpha}^{2}\left(K_{E}-6 K_{F}\right)-K_{E}}\left(\frac{\partial \vartheta_{\alpha}}{\partial \alpha}\right)^{2}\right. \\
& \left.\left.+\frac{3 / 4 \rho_{\alpha}^{2}}{\left(1-3 / 4 \rho_{\alpha}^{2}\right)\left[8 K_{F}+\rho_{\alpha}^{2}\left(K_{E}-6 K_{F}\right)\right]}\left(\frac{\partial \rho_{\alpha}}{\partial \alpha}\right)^{2}\right]\right\} \mathrm{d} A,
\end{aligned}
$$

where $K_{S}, K_{E}$, and $K_{F}$ are the scaled macro-stiffnesses corresponding to the micro-stiffnesses $k_{S}, k_{E}$, and $k_{F}$, respectively.

The values of these macro-stiffnesses were calibrated by fitting total reaction force data (Figure 5) and six discrete displacements obtained by local digital image correlation (DIC) [56] in the considered bias extension test for global longitudinal strains up to ca. $25 \%$. A very good agreement is observed over the whole range of investigated strains. 


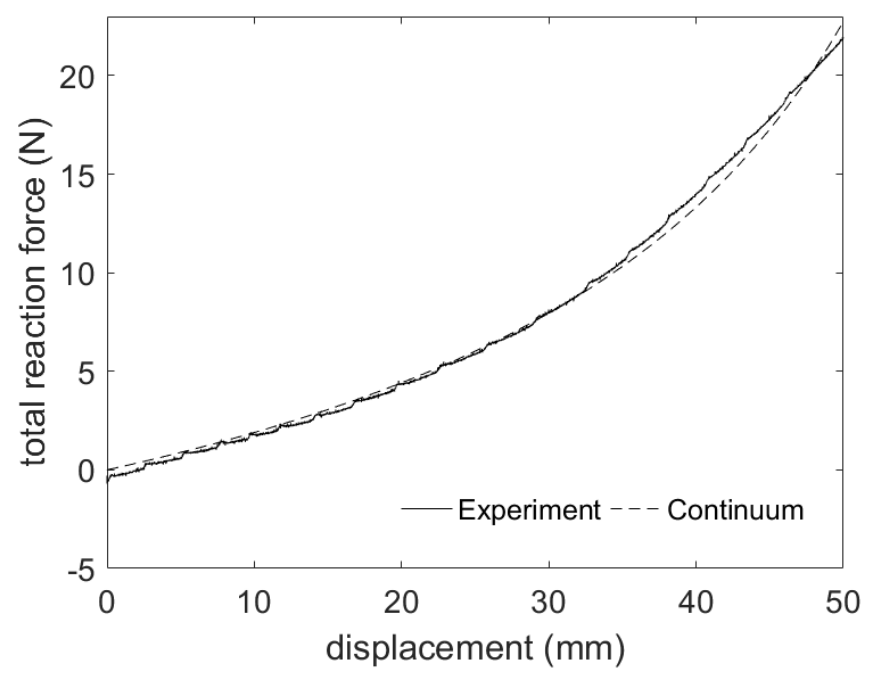

Figure 5: Reaction force vs. prescribed displacement for total longitudinal strains up to ca. $25 \%$.

The calibrated parameters are reported in Table 1). They are the only ones that will be used in the sequel for validation purposes.

Table 1: Calibrated parameters of the continuum model.

\begin{tabular}{lll}
\hline$K_{F}$ & $K_{E}$ & $K_{S}$ \\
\hline $0.9 \mathrm{~J}$ & $0.33 \mathrm{~J}$ & $34 \mathrm{~N} \cdot \mathrm{m}^{-1}$
\end{tabular}

Solving the weak or strong form problems for the homogenized continuum model is a direct problem in the sense that, given boundary conditions on a reference domain, the admissible displacement field that makes stationary the energy functional is sought. The solution of the weak form was implemented within the commercial software COMSOL Multiphysics. In the last years, thanks to the interest in gradient elasticity, efforts in numerically addressing such non-standard problems have grown, thus establishing well-known methodologies [28, 29 . 
It is worth noting that besides the term $\partial \vartheta_{\alpha} / \partial \alpha$ (i.e., curvature of material ${ }_{235}$ lines along $\mathbf{e}_{\alpha}$ in the reference configuration), the term $\partial \rho_{\alpha} / \partial \alpha$ also appears in Equation (19), which is the derivative along the direction $\mathbf{e}_{\alpha}$ of the stretch of material lines along $\mathbf{e}_{\alpha}$ in the reference configuration. This observation implies that, for the above continuum deformation energy, conditions on the boundaries (Figure 3(a)) are prescribed on i) the normal displacement gradient, ii) the displacement itself, and conditions at the vertices on the displacement [57. For instance, in the considered bias extension test, the reference domain $\Omega$ is nominally subjected to the essential boundary conditions described in Table 2 .

Table 2: Nominal boundary conditions for bias extension test of bi-pantgraphic fabrics. The vector $\mathbf{n}$ is the outward unit normal to $\partial \Omega$ in $\mathbf{x}$ (see Figure 3 a)).

\begin{tabular}{ll}
\hline$\partial \Omega_{1}$ & $\partial \Omega_{3}$ \\
\hline $\mathbf{u}_{H C}(\mathbf{x})=\mathbf{0}$ & $\mathbf{u}_{H C}(\mathbf{x})=\bar{u} \mathbf{e}_{\zeta}, \bar{u} \in \mathbb{R}^{+}$ \\
$\nabla \mathbf{u}_{H C}(\mathbf{x}) \cdot \mathbf{n}(\mathbf{x})=0$ & $\nabla \mathbf{u}_{H C}(\mathbf{x}) \cdot \mathbf{n}(\mathbf{x})=0$
\end{tabular}

The kinematic prescriptions in the second row, as combined with those in the first row of Table 2 imply that $\nabla \mathbf{u}_{H C}(\mathbf{x})=\mathbf{0}$ on $\Omega_{1} \cup \Omega_{3}$, which is consistent 245 with the micro-macro identification procedure when using stocky rhomboidal elements (Figure 2).

\section{Bias Extension Test}

\subsection{Experimental Configuration}

A displacement-controlled bias extension test was performed on the sample shown in Figure 2 for macroscopic strains up to ca. $40 \%$ (Figure 6). In textile mechanics, the bias extension test is a standard experiment to investigate inplane combined shear and tensile responses of materials made up of two families of fibers [58]. The bias extension test is performed on samples having the shape of a rectangle with dimension in the loading direction greater than the width [59]. 
In a bias extension test, the fibers (i.e., pantographic ones in the present study) are initially oriented at $\pm 45^{\circ}$ with respect to the loading direction. In other words, the sample to be used in a bias extension test is cut along the two biases, i.e., the two orthogonal directions forming $\pm 45^{\circ}$ with the fibers. An MTS Tytron 250 testing-device prescribed longitudinally (i.e., on the right side of the specimen) an increasing displacement with a quasi-static loading rate of $15 \mathrm{~mm} / \mathrm{min}$. Pictures of the surface during deformation were acquired $(0.5 \mathrm{fps}$, i.e., nominally for $1 \mathrm{~mm}$ displacement increments) by means of a Canon EOS $600 \mathrm{D}$ camera with a definition of $4272 \times 2848$ pixels and an 8 -bit dynamic range.

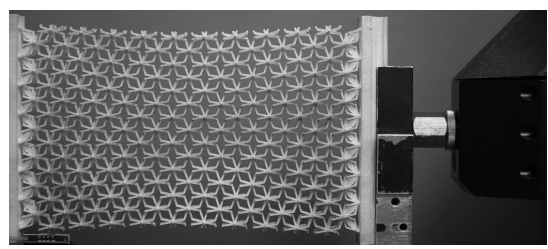

(a) \#24

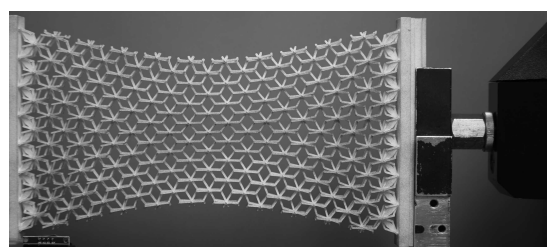

(c) $\# 70$

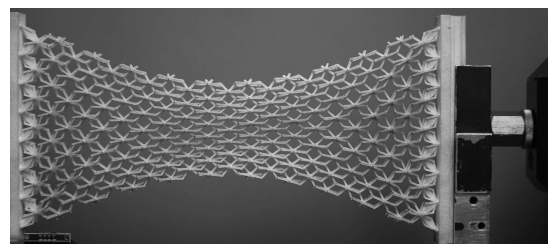

(e) \#116

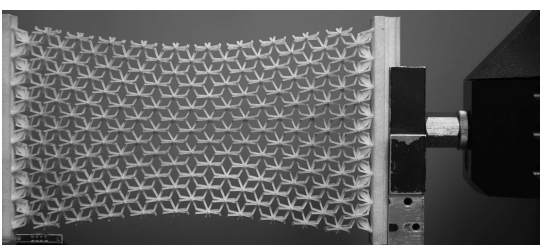

(b) $\# 47$

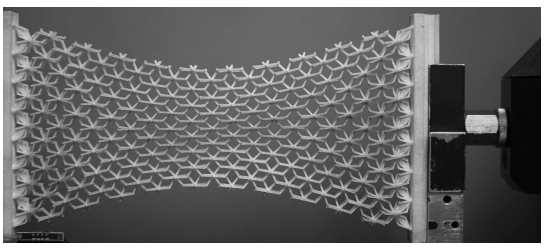

(d) \#93

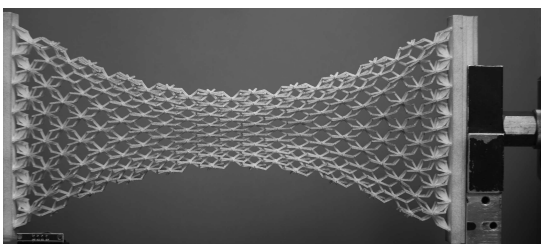

(f) \#139

Figure 6: Pictures of deformed configurations in a bias extension test on bi-pantographic metamaterial.

Bi-pantographic fabrics exhibit an extremely wide elastic range (i.e., the dissipated energy is negligibly small compared to the elastic energy stored 
during loading). Such extreme elastic strains are achievable as their macroscopic levels are considerably greater than single-elastic-element strains. While satisfying boundary conditions and internal connection constraints, elements arrange locally in space to minimize the total deformation energy by mimicking mechanisms corresponding to zero-energy deformation modes [49].

\subsection{FE-Based Digital Image Correlation}

The 139 pictures of deformed configurations were registered with the picture of the reference configuration (Figure 7) via Digital Image Correlation (DIC [60, 61]). Various approaches have been introduced, namely, local (i.e., 275 subset-based) analyses [62, 63, 64], and global (e.g., finite element based) techniques [53, 54, 55]. In the present case, FE-DIC was used to measure displacement fields since it provides a very natural link with finite element simulations (i.e., the meshes can be made identical provided the element size is compatible with measurement uncertainties [65]). Working within the high-deformation regime in the considered bias extension test, bi-pantographic specimens undergo extremely large contraction-to-extension ratios equal to ca. 0.85. Consequently, a solution will be sought very far from the reference configuration, which adds complexity for both modeling and DIC approaches.

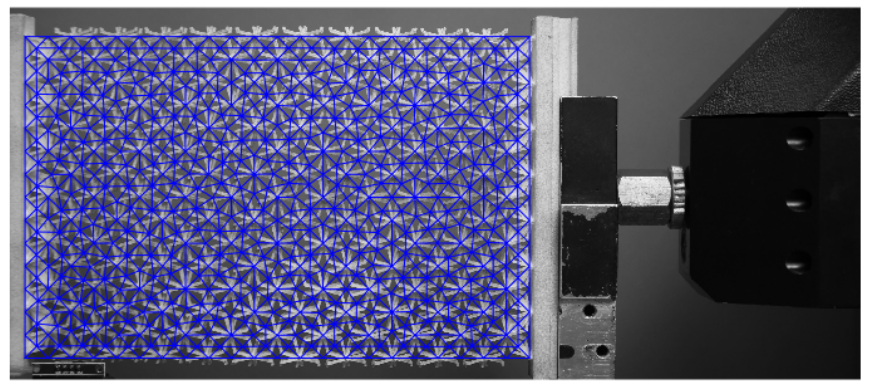

Figure 7: Picture of the reference configurations in a bias extension test on bi-pantographic metamaterial. The mesh used in the simulations of the $\mathrm{HC}$ model is overlaid. Its characteristic size is equal to 34 pixels $(\approx 2.7 \mathrm{~mm})$.

In global DIC [55, 66], two gray level images $f$ and $g$ are registered by 
minimizing the global residual

$$
\Phi_{c}^{2}=\sum_{\mathrm{ROI}} \rho_{D I C}^{2}(\mathbf{x})
$$

which is the integration over the region of interest (ROI) of the squared sum of the gray level residual

$$
\rho_{D I C}(\mathbf{x})=f(\mathbf{x})-g\left(\mathbf{x}+\mathbf{u}_{D I C}(\mathbf{x})\right)
$$

that is computed for each pixel $\mathbf{x}$ of the ROI, and corresponds to the difference of the gray levels in the reference configuration $f$ and that in the deformed configuration $g$ corrected by the measured displacement $\mathbf{u}_{D I C}(\mathbf{x})$. The latter is parameterized with a set of degrees of freedom $v_{n}$, which are gathered in the column vector $\{\boldsymbol{v}\}$

$$
\mathbf{u}_{D I C}(\mathbf{x})=\sum_{n} v_{n} \boldsymbol{\psi}_{n}(\mathbf{x})
$$

associated with the trial fields $\boldsymbol{\psi}_{n}$. The minimization with respect to the unknown degrees of freedom is nonlinear. One way of performing such 295 minimization is to follow modified Newton schemes [67, 66]. In the sequel, meshes made of $\mathrm{T} 3$ elements were considered [68. The trial displacement fields then become the shape functions of T3 elements. Very recently, T3-DIC was shown to be applicable to pantographic metamaterials at various scales of kinematic descriptions [46, 47, 48, 52].

With the studied material, the speckle pattern was only applied over a small area of the ROI (Figure 7). In order to properly converge, due to such a speckle pattern, elastic regularization was considered in the global DIC approach [69, 70]. A second term was added to the global residual $\Phi_{c}^{2}$, in the spirit of Tikhonov and Arsenin regularization schemes [71]. In the present case, the equilibrium gap functional [72] was selected for inner nodes and boundary nodes that were traction-free

$$
\Phi_{m}^{2}=\{\boldsymbol{v}\}^{\top}[\mathbf{K}]^{\top}[\mathbf{K}]\{\boldsymbol{v}\}
$$

where $[\mathbf{K}]$ is the rectangular stiffness matrix restricted to the selected nodes. 
For the other edge nodes, a similar penalization was considered

$$
\Phi_{b}^{2}=\{\boldsymbol{v}\}^{\top}[\mathbf{L}]^{\top}[\mathbf{L}]\{\boldsymbol{v}\}
$$

where $[\mathbf{L}]$ is a second order operator restricted to edge nodes $[73$. The global residual to be minimized then consists of the weighted sum of the previous three quantities, namely, $\Phi_{c}^{2}, \Phi_{m}^{2}$ and $\Phi_{b}^{2}$. Since the physical dimension of the correlation functional is different from that of the other two functionals, they need to be made dimensionless. Penalization weights premultiplying $\Phi_{m}^{2}$ and $\Phi_{b}^{2}$ are introduced [69, 70, 173]. They are proportional to regularization lengths raised to the fourth power. The larger the regularization lengths, the more weight is put on the penalty terms. This penalization acts as a low-pass mechanical filter, namely, all high frequency components of the displacement field that are not mechanically admissible are filtered out. Similarly, for low-contrast areas mechanical regularization provides the displacement interpolation.

In the present case, the regularization length was set to 150 pixels, which is the size of elementary cells (Figure $3(\mathrm{~b})$ ). It is worth noting that other choices could have been made, in particular, smaller regularization lengths. However, they would no longer represent a physical length but rather a help 325 for convergence of the minimization scheme. Consequently, such choices will not be discussed hereafter. Similarly, analyses at lower scales [47, 48, 52] were not considered to remain consistent with the modeling framework of Section 2 . Further, since large strain levels occur, the previous regularization was only applied to incremental displacements $\{\delta \boldsymbol{v}\}$ (i.e., from one picture to the next)

$$
\Phi_{m}^{2}=\{\delta \boldsymbol{v}\}^{\top}[\mathbf{K}]^{\top}[\mathbf{K}]\{\delta \boldsymbol{v}\}
$$

330 and

$$
\Phi_{b}^{2}=\{\delta \boldsymbol{v}\}^{\top}[\mathbf{L}]^{\top}[\mathbf{L}]\{\delta \boldsymbol{v}\}
$$

Such regularization leads to assume Hencky-type elasticity at the scale of the regularization length, which is less stringent than infinitesimal elastic in a situation of finite strains. 
When dealing with large deformations, the solution for the current picture can be initialized from the solution of the previous step. A further approach can be adopted, namely incremental DIC (as opposed to direct DIC, i.e., the approach presented thus far where the reference picture is not the initial one). In this approach, in a step-wise procedure, the picture preceding the current one is chosen as the reference picture for the correlation analysis. Consequently, 340 the mesh is gradually deformed to follow the motions. The incremental displacements are cumulated to determine the overall (Lagrangian) displacement fields with respect to the non-updated initial picture. As the minimization problem for incremental DIC does not have an optimal substructure property, such cumulated displacements are different from those obtained by direct DIC 345 and may yield higher cost functions, i.e., higher global residuals (Equation (23)). For pantographic structures, this may not be the case, and needs to be checked. Last, the displacement results obtained by incremental DIC are exploited as initialization to direct DIC for the corresponding picture pairs.

Figure 8 shows the change of root mean square residuals, i.e., RMS $\left(\rho_{D I C}\right)$, for direct and incremental DIC when using the discretization shown in Figure 7 . In the present case, direct DIC leads to lower residuals in comparison with incremental DIC. The former will be selected when comparing the measurements with numerical simulations. The fact that the residual levels increase as the bi-pantographic fabric is deformed more is an indication that a continuum description is no longer able to fully capture the details of the experimental kinematics. This observation is not a shortcoming of DIC but rather a check on the kinematic hypotheses made to capture the full complexity of the test via macroscale quantities. 


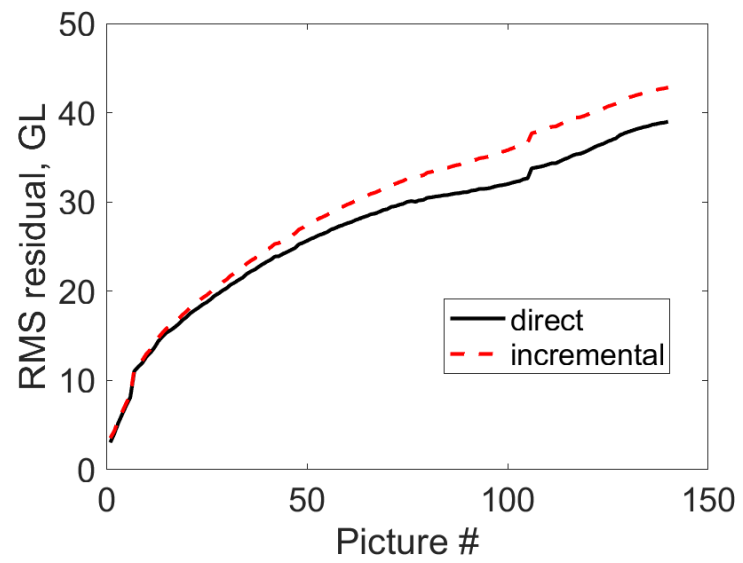

Figure 8: RMS gray level correlation residual for direct and incremental DIC using the mesh shown in Figure 7

The effect of the discretization has also to be assessed on the previous results.

360 Three additional regular meshes were considered with different characteristic length scales ranging from 77 to 24 pixels. The latter is defined as the mean square root of element area. The mesh shown in Figure 7 has a characteristic length of 34 pixels. In Figure 9, the RMS residuals are reported for the four different discretizations. Mesh convergence is observed for the three finer discretizations. This result is related to the selected regularization length (here equal to 150 pixels), which controls the high frequency fluctuations of displacement fields. 


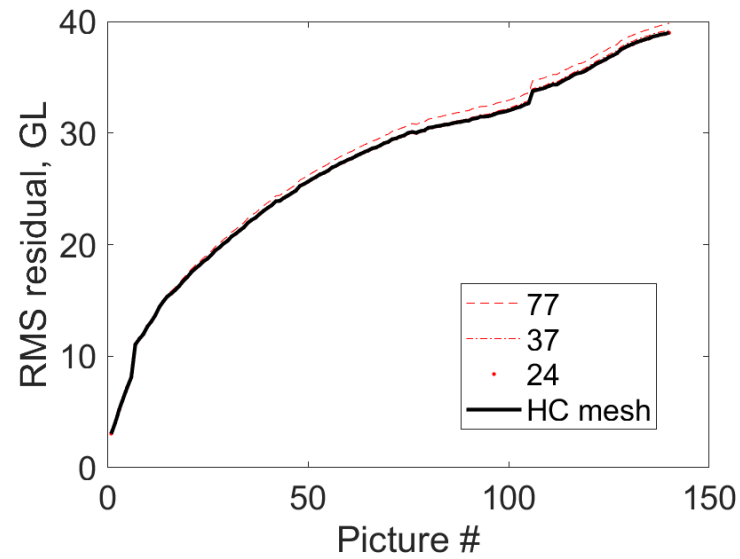

Figure 9: RMS gray level residuals for direct DIC with different meshes.

From all these last results, it is concluded that the finite element mesh used in the numerical simulations (Figure 7) is also compatible with regularized DIC measurements at the macroscopic scale. With the selected DIC parameters the noise-floor displacement uncertainty is less than to 0.025 pixel (i.e., $2 \mu \mathrm{m}$ ), which is very small given the large deformations experienced by the studied metamaterial.

\section{Validation Results}

375 When quantitatively comparing the performances of DIC and a homogenized continuum model, a measure of goodness of kinematic results has to be defined given the fact that the load response was used in the calibration procedure (see Figure 5). The first (and standard) way of comparing DIC measurements with models is to report the displacement fields $\mathbf{u}_{D I C}$ and $\mathbf{u}_{H C}$ over the meshed ROI as obtained for, say, picture \#93 (Figure 10). It may be concluded that the two results are qualitatively in agreement. 


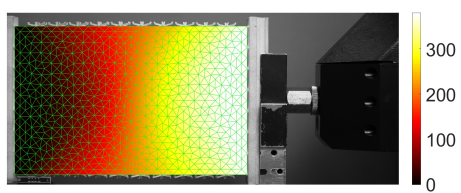

(a) DIC, \#93

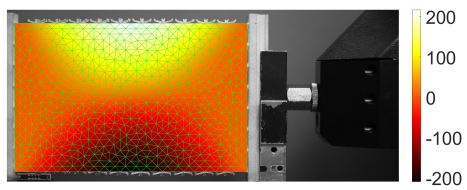

(c) DIC, $\# 93$

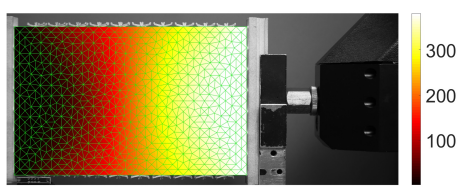

(b) $\mathrm{HC}, \# 93$

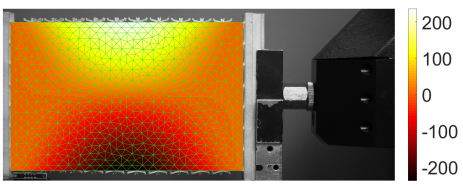

(d) HC, \#93

Figure 10: Displacement fields $\mathbf{u}_{D I C}$ and $\mathbf{u}_{H C}$ (longitudinal component along $\mathbf{e}_{\zeta}$ on top, transverse component along $\mathbf{e}_{\varsigma}$ at bottom) expressed in pixels over the meshed ROI as obtained for picture \#93 by means of the validation procedure shown in Figure 1 The reference picture lies in the background and the finite element mesh in the foreground.

To get a more quantitative assessment, displacement differences should be computed [74]. In the present case, since the same mesh was used, this comparison is straightforward as it can be performed node-wise. Figure 11 shows the displacement difference fields for the same analyzed picture. It is worth noting that, despite the fact that displacements measured by DIC at short sides of the specimen were used as boundary condition for the continuum model, the displacement difference between the latter and DIC is non-zero along such short boundaries (see Figure 11). The continuum model was numerically solved by means of a mixed weak finite element formulation in which boundary conditions were not encoded strongly, i.e., node-wise within test functions, but were enforced weakly via Lagrange multipliers. Therefore, such discrepancy should be expected. In particular, this discrepancy is observed at corners and is concentrated in the two triangular mesh elements intersecting at a corner. It is expected that such a discrepancy will be confined to ever smaller regions when 
the mesh size decreases.

The RMS difference for the longitudinal component is equal to 8 pixels, and 9 pixels in the transverse direction. These levels are two orders of magnitude higher than the measurement uncertainty for both components. They may seem very high. However, it is worth noting that the longitudinal displacement range is of the order of 400 pixels. It is therefore concluded that the observed differences are related to model errors. Yet, it cannot be decided which part of the data, namely, experimental and/or numerical quantities are questionable.

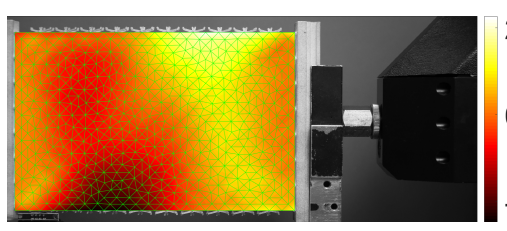

(a)

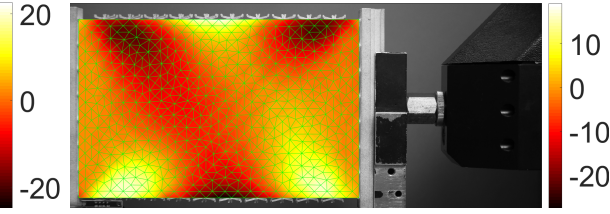

(b)

Figure 11: Displacement difference field $\mathbf{u}_{H C}-\mathbf{u}_{D I C}$ (longitudinal component along $\mathbf{e}_{\zeta}$ (a) and transverse component along $\left.\mathbf{e}_{\varsigma}(\mathrm{b})\right)$ expressed in pixels over the meshed ROI as obtained for picture \#93 by means of the validation procedure shown in Figure 1 The reference picture lies in the background and the finite element mesh in the foreground.

A second route consists in computing the gray level residuals associated with numerical simulations [50]. This procedure is viable provided the measured boundary conditions are prescribed to the numerical simulations (see Figure 1). It was shown to be the best in various experimental configurations [75, 51]. It will also be utilized herein. By following such path, the merits of each approach are independently probed against the experimental video. The correlation residual becomes a pixel-wise measure of goodness, and its RMS computed over the ROI a global goodness measure. Gray level residuals were defined in Equation (24) for DIC. The same equation, substituting $\mathbf{u}_{H C}$ for $\mathbf{u}_{D I C}$ will be considered for evaluating the gray level residuals associated with the homogenized continuum model

$$
\rho_{F E}(\mathbf{x})=f(\mathbf{x})-g\left(\mathbf{x}+\mathbf{u}_{F E}(\mathbf{x})\right)
$$


Figure 12 shows the residual maps corresponding to the displacement fields measured via direct DIC and predicted with the HC model (Figure 10). The residuals are very low close to the left and right edges since the sample does not deform much and the measured displacement is consistent with the experiment. This observation applies for both approaches since the HC simulations were driven by measured Dirichlet boundary conditions. Even if imperfectly prescribed (see above discussion), the residuals are very low in those areas, thereby proving that the measured and simulated displacements are trustworthy.

Conversely, the residuals are higher in the central part of the sample for $\mathrm{HC}$ 425 simulations compared with DIC measurements. On a more quantitative basis, the RMS residual is equal to 31 gray levels for DIC and 43 gray levels for HC. These levels are significantly higher than those observed at the beginning of the experiment (i.e., $\approx 3$ gray levels), which are close to acquisition noise. This result shows that the present model, even though in very good agreement with force measurements (Figure 5) is not able to fully capture the local complexities of the experimental kinematics. This conclusion also applies (to a lesser degree) to DIC results whose kinematic assumptions are not fully capturing the complexity of the local deformation of the studied metamaterial.

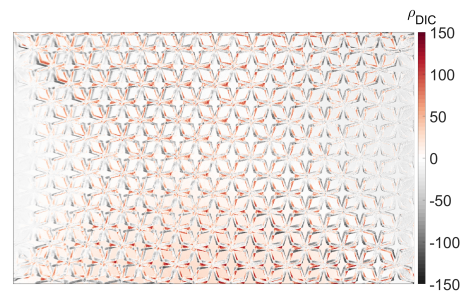

(a) DIC

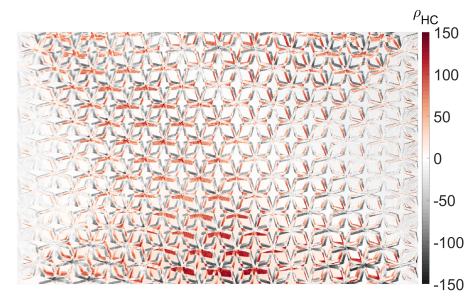

(b) $\mathrm{HC}$

Figure 12: Gray level residual maps for direct DIC and HC predictions corresponding to picture \#93.

The same type of analysis can be repeated for any of the 139 pictures acquired during the test. Only 6 of them are reported in the Appendix (see 
Figures 14 and 15). They correspond to the deformed configurations shown in Figure 6. For comparison purposes, the residual maps of the first picture are shown as well. They are very low and very close to the acquisition noise of the camera. For both DIC and HC modeling, the absolute level of the 440 pixel-wise correlation residuals increases with the picture number. Further, in the correlation residuals the microscale pattern emerges, which at the considered kinematic description scale, is not fully captured. This observation indicates that the results for DIC and HC may be improved by lower-scale analyses [47, 48, 52].

${ }_{445}$ A plot of the RMS residuals versus picture number for direct DIC, incremental DIC and HC simulations is shown in Figure 13. DIC performs better than the $\mathrm{HC}$ model, be it direct or incremental. This trend is to be expected since DIC, contrary to HC modeling, mainly minimizes the correlation residuals (see summand in Equation (23)). For all approaches, the RMS residuals increase 450 with the picture number and their relative increment is decreasing. Further, the residuals of the $\mathrm{HC}$ model are not too high in comparison with those of DIC, which allows the present macroscopic model to be deemed reasonable. Last, three jumps are observed (for pictures \#7, 106, 128), whose occurrence is due to changes in lighting conditions. 


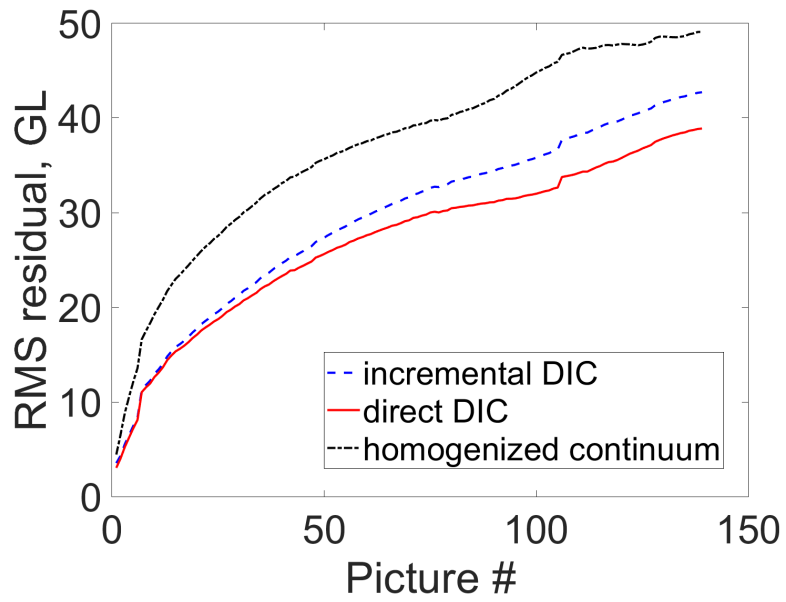

Figure 13: Gray level RMS residuals for direct DIC, incremental DIC, and HC simulations.

\section{Conclusion}

In this paper, a first comparison between performances of FE-based DIC and a homogenized continuum model was performed within the finite strain regime of a recently designed metamaterial with bi-pantographic microstructure. The homogenized model was derived from a discrete description at the microscale.

${ }_{460}$ Experimental displacement data (not full-fields) up to ca. $25 \%$ total elongations were used for calibrating the model parameters. In the present case, the same FE discretization could be used thanks to Hencky-type elastic regularization (at the scale of the metamaterial cells) of DIC.

A measure of goodness of kinematic results was devised for both approaches, 465 which allows the merits and shortcomings of DIC and HC simulations to be probed in an absolute (i.e., independent) way via gray level residuals. It was observed that, while DIC performed better than the continuum model, the latter gave good results given the very high levels of strains. This result thus shows that the homogenized model can capture the main deformation features but not

470 the complexity of local details. The constitutive law is not only descriptive but also predictive for a wider range than that used for calibration purposes (the 
analysis was conducted up to ca. $40 \%$ macroscopic elongation).

Possible outlooks include the combination of the two methodologies in so-called integrated DIC [68, 76, and especially an automated calibration

475 procedure of the $\mathrm{HC}$ model based on numerical sensitivity analyses [77] in, e.g., , bias extension tests. Such procedure will aim at minimizing the gray level residuals under the constraint of mechanical admissibility provided by numerical simulations using the HC model or any other Ansatz.

\section{Acknowledgments}

${ }_{480}$ The authors thank P. Seppecher and G. Ganzosch for stimulating discussions. 


\section{References}

[1] R. A. Toupin, Theories of elasticity with couple-stress, Archive for Rational Mechanics and Analysis 17 (2) (1964) 85-112.

[2] A. Green, Micro-materials and multipolar continuum mechanics, International Journal of Engineering Science 3 (5) (1965) 533-537.

[3] R. D. Mindlin, Second gradient of strain and surface-tension in linear elasticity, International Journal of Solids and Structures 1 (4) (1965) 417438.

[4] G. A. Maugin, Generalized continuum mechanics: what do we mean by that?, in: Mechanics of Generalized Continua, Springer, 2010, pp. 3-13.

[5] F. dell'Isola, T. Lekszycki, M. Pawlikowski, R. Grygoruk, L. Greco, Designing a light fabric metamaterial being highly macroscopically tough under directional extension: first experimental evidence, Zeitschrift für angewandte Mathematik und Physik 66 (2015) 3473-3498.

${ }_{495}[6]$ F. dell'Isola, I. Giorgio, M. Pawlikowski, N. L. Rizzi, Large deformations of planar extensible beams and pantographic lattices: heuristic homogenization, experimental and numerical examples of equilibrium, Proc. R. Soc. A 472 (2185) (2016).

[7] J.-J. Alibert, P. Seppecher, F. dell'Isola, Truss modular beams with deformation energy depending on higher displacement gradients, Mathematics and Mechanics of Solids 8 (1) (2003) 51-73.

[8] H. Abdoul-Anziz, P. Seppecher, Strain gradient and generalized continua obtained by homogenizing frame lattices, Mathematics and Mechanics of Complex Systems (2018).

505 [9] E. Turco, F. dell'Isola, N. L. Rizzi, R. Grygoruk, W. H. Müller, C. Liebold, Fiber rupture in sheared planar pantographic sheets: numerical and 
experimental evidence, Mechanics Research Communications 76 (2016) 8690.

[10] E. Turco, M. Golaszewski, A. Cazzani, N. Rizzi, Large deformations induced in planar pantographic sheets by loads applied on fibers: experimental validation of a discrete lagrangian model, Mechanics Research Communications 76 (2016) 51-56.

[11] E. Turco, K. Barcz, M. Pawlikowski, N. Rizzi, Non-standard coupled extensional and bending bias tests for planar pantographic lattices. part i: numerical simulations, Zeitschrift für angewandte Mathematik und Physik 67 (5) (2016) 122.

[12] E. Turco, N. Rizzi, Pantographic structures presenting statistically distributed defects: numerical investigations of the effects on deformation fields, Mechanics Research Communications 77 (2016) 65-69.

[13] E. Turco, I. Giorgio, A. Misra, F. dell'Isola, King post truss as a motif for internal structure of (meta) material with controlled elastic properties, Royal Society Open Science 4 (10) (2017).

[14] E. Turco, A. Misra, M. Pawlikowski, F. dell'Isola, F. Hild, Enhanced piola-hencky discrete models for pantographic sheets with pivots without deformation energy: Numerics and experiments, International Journal of Solids and Structures (2018).

[15] U. Andreaus, M. Spagnuolo, T. Lekszycki, S. R. Eugster, A ritz approach for the static analysis of planar pantographic structures modeled with nonlinear euler-bernoulli beams, Continuum Mechanics and Thermodynamics (2018) 1-21.

[16] D. Steigmann, M. Faulkner, Variational theory for spatial rods, Journal of Elasticity 33 (1) (1993) 1-26. 
[17] A. Cazzani, M. Malagù, E. Turco, F. Stochino, Constitutive models for strongly curved beams in the frame of isogeometric analysis, Mathematics and Mechanics of Solids 21 (2) (2016) 182-209.

[18] A. Cazzani, M. Malagù, E. Turco, Isogeometric analysis: a powerful numerical tool for the elastic analysis of historical masonry arches, Continuum Mechanics and Thermodynamics 28 (1-2) (2016) 139-156.

[19] M. Spagnuolo, U. Andreaus, A targeted review on large deformations of planar elastic beams: extensibility, distributed loads, buckling and post-

प buckling, Mathematics and Mechanics of Solids (2018). doi:10.1177/ 1081286517737000 .

[20] H. Altenbach, V. Eremeyev, On the linear theory of micropolar plates, ZAMM-Journal of Applied Mathematics and Mechanics/Zeitschrift für Angewandte Mathematik und Mechanik 89 (4) (2009) 242-256.

[21] V. A. Eremeyev, L. P. Lebedev, H. Altenbach, Foundations of micropolar mechanics, Springer Science \& Business Media, 2012.

[22] H. Altenbach, M. Bîrsan, V. A. Eremeyev, Cosserat-type rods, in: Generalized Continua from the Theory to Engineering Applications, Springer, 2013, pp. 179-248.

[23] V. A. Eremeyev, F. dell'Isola, C. Boutin, D. Steigmann, Linear pantographic sheets: existence and uniqueness of weak solutions, Journal of Elasticity (2017) 1-22.

[24] L. Placidi, A. El Dhaba, Semi-inverse method à la saint-venant for two-dimensional linear isotropic homogeneous second-gradient elasticity, Mathematics and Mechanics of Solids 22 (5) (2017) 919-937.

[25] L. Placidi, U. Andreaus, A. Della Corte, T. Lekszycki, Gedanken experiments for the determination of two-dimensional linear second gradient elasticity coefficients, Zeitschrift für angewandte Mathematik und $560 \quad$ Physik 66 (6) (2015) 3699-3725. 
[26] L. Placidi, U. Andreaus, I. Giorgio, Identification of two-dimensional pantographic structure via a linear $\mathrm{d} 4$ orthotropic second gradient elastic model, Journal of Engineering Mathematics (2016) 1-21.

[27] B. E. Abali, W. H. Müller, V. A. Eremeyev, Strain gradient elasticity with geometric nonlinearities and its computational evaluation, Mechanics of Advanced Materials and Modern Processes 1 (1) (2015) 4.

[28] J. Niiranen, S. Khakalo, V. Balobanov, A. H. Niemi, Variational formulation and isogeometric analysis for fourth-order boundary value problems of gradient-elastic bar and plane strain/stress problems, Computer Methods in Applied Mechanics and Engineering 308 (2016) 182211.

[29] S. Khakalo, J. Niiranen, Isogeometric analysis of higher-order gradient elasticity by user elements of a commercial finite element software, Computer-Aided Design 82 (2017) 154-169.

${ }_{575}[30]$ V. Balobanov, J. Kiendl, S. Khakalo, J. Niiranen, Kirchhoff-love shells within strain gradient elasticity: Weak and strong formulations and an h3conforming isogeometric implementation, Computer Methods in Applied Mechanics and Engineering 344 (2019) 837-857.

[31] L. Placidi, L. Greco, S. Bucci, E. Turco, N. Rizzi, A second gradient formulation for a $2 \mathrm{~d}$ fabric sheet with inextensible fibres, Zeitschrift für angewandte Mathematik und Physik 67(5) (114) (2016).

[32] E. Barchiesi, M. Spagnuolo, L. Placidi, Mechanical metamaterials: a state of the art, Mathematics and Mechanics of Solids (2018).

[33] F. dell'Isola, P. Seppecher, J. J. Alibert, T. Lekszycki, R. Grygoruk, M. Pawlikowski, D. Steigmann, I. Giorgio, U. Andreaus, E. Turco, et al., Pantographic metamaterials: an example of mathematically driven design and of its technological challenges, Continuum Mechanics and Thermodynamics 31 (4) (2019) 851-884. 
[34] M. Spagnuolo, P. Peyre, C. Dupuy, Phenomenological aspects of quasi-

[35] F. dell'Isola, E. Turco, A. Misra, Z. Vangelatos, C. Grigoropoulos, V. Melissinaki, M. Farsari, Force-displacement relationship in micro-metric pantographs: Experiments and numerical simulations, Comptes Rendus

[40] A. Misra, P. Poorsolhjouy, Granular micromechanics model for damage and plasticity of cementitious materials based upon thermomechanics, Mathematics and Mechanics of Solids (2015).

[41] A. Misra, V. Singh, Thermomechanics-based nonlinear rate-dependent coupled damage-plasticity granular micromechanics model, Continuum Mechanics and Thermodynamics 27 (4-5) (2015) 787. 
[42] M. De Angelo, M. Spagnuolo, F. D'annibale, A. Pfaff, K. Hoschke, A. Misra, C. Dupuy, P. Peyre, J. Dirrenberger, M. Pawlikowski, The macroscopic behavior of pantographic sheets depends mainly on their microstructure: experimental evidence and qualitative analysis of damage in metallic specimens, Continuum Mechanics and Thermodynamics (2019) 1-23.

[43] N. Engheta, R. Ziolkowski, Metamaterials: Physics and Engineering Explorations, John Wiley \& Sons, Inc., 2006.

[44] A. Misra, T. Lekszycki, I. Giorgio, G. Ganzosch, W. H. Müller, F. dell'Isola, Pantographic metamaterials show atypical poynting effect reversal, Mech. Res. Commun. 89 (2018) 6-10.

[45] M. Spagnuolo, K. Barcz, A. Pfaff, F. dell'Isola, P. Franciosi, Qualitative pivot damage analysis in aluminum printed pantographic sheets: numerics and experiments, Mechanics Research Communications 83 (2017) 47-52.

[46] E. Turco, A. Misra, M. Pawlikowski, F. dell'Isola, F. Hild, Enhanced Piola-Hencky discrete models for pantographic sheets with pivots without deformation energy: Numerics and experiments, International Journal of Solids and Structures 147 (2018) 94-109.

[47] F. dell'Isola, P. Seppecher, J.-J. Alibert, T. Lekszycki, R. Grygoruk, M. Pawlikowski, D. Steigmann, I. Giorgio, U. Andreaus, E. Turco, M. Golaszewski, N. Rizzi, C. Boutin, V. Eremeyev, A. Misra, L. Placidi, E. Barchiesi, L. Greco, M. Cuomo, A. Cazzani, A. Corte, A. Battista, D. Scerrato, I. Zurba Eremeeva, Y. Rahali, J.-F. Ganghoffer, W. Müller, G. Ganzosch, M. Spagnuolo, A. Pfaff, K. Barcz, K. Hoschke, J. Neggers, F. Hild, Pantographic metamaterials: an example of mathematically driven design and of its technological challenges, Continuum Mechanics and Thermodynamics 31 (4) (2019) 851-884.

[48] F. dell'Isola, P. Seppecher, M. Spagnuolo, E. Barchiesi, F. Hild, T. Lekszycki, I. Giorgio, L. Placidi, U. Andreaus, M. Cuomo, 
S. Eugster, A. Pfaff, K. Hoschke, R. Langkemper, E. Turco, R. Sarikaya, A. Misra, M. De Angelo, F. D'Annibale, A. Bouterf, X. Pinelli, A. Misra, B. Desmorat, M. Pawlikowski, C. Dupuy, D. Scerrato, P. Peyre, M. Laudato, L. Manzari, P. Göransson, C. Hesch, S. Hesch, P. Franciosi, J. Dirrenberger, F. Maurin, Z. Vangelatos, C. Grigoropoulos, ${ }_{650}$ V. Melissinaki, M. Farsari, W. Muller, E. Abali, C. Liebold, G. Ganzosch, P. Harrison, R. Drobnicki, L. Igumnov, F. Alzahrani, T. Hayat, Advances in Pantographic Structures: Design, Manufacturing, Models, Experiments and Image Analyses, Continuum Mechanics and Thermodynamics 31 (4) (2019) 1231-1282.

[49] P. Seppecher, J.-J. Alibert, F. D. Isola, Linear elastic trusses leading to continua with exotic mechanical interactions, in: Journal of Physics: Conference Series, Vol. 319, IOP Publishing, 2011, p. 012018.

[50] M. B. A. Buljac, M. Shakoor, P.-O. Bouchard, T. Morgeneyer, F. Hild, Numerical validation framework for micromechanical simulations based on $660 \quad$ synchrotron 3D imaging, Comput. Mech. 59 (3) (2017) 419-441.

[51] M. Shakoor, A. Buljac, J. Neggers, F. Hild, T. Morgeneyer, L. Helfen, M. Bernacki, P.-O. Bouchard, On the choice of boundary conditions for micromechanical simulations based on 3D imaging, International Journal of Solids and Structures 112 (2017) 83-96.

665 [52] F. Hild, A. Misra, F. dell'Isola, Multiscale DIC applied to Pantographic Structures, Experimental Mechanics (10.1007/s11340-020-00636-y) (2020).

[53] G. Broggiato, Adaptive image correlation technique for full-field strain measurement, in: C. Pappalettere (Ed.), 12th Int. Conf. Exp. Mech., McGraw Hill, Lilan (Italy), 2004, pp. 420-421.

${ }_{670}^{6}$ [54] Y. Sun, J. Pang, C. Wong, F. Su, Finite-element formulation for a digital image correlation method, Appl. Optics 44 (34) (2005) 7357-7363. 
[55] G. Besnard, F. Hild, S. Roux, "Finite-element" displacement fields analysis from digital images: Application to Portevin-Le Chatelier bands, Exp. Mech. 46 (2006) 789-803.

675 [56] E. Barchiesi, Synthesis of second gradient architectured metamaterials: Micro-to-macro approaches, numerical investigations, additive manufacturing and experimental validation, $\mathrm{PhD}$ thesis, Sapienza University of Rome (Italy) (2019).

[57] N. Auffray, F. dell'Isola, V. Eremeyev, A. Madeo, G. Rosi, Analytical continuum mechanics à la Hamilton-Piola least action principle for second gradient continua and capillary fluids, Mathematics and Mechanics of Solids 20 (4) (2015) 375-417.

[58] D. Cooper, A bias extension test, Textile Research Journal 33 (4) (1963) $315-317$.

685 [59] P. Boisse, N. Hamila, E. Guzman Maldonado, A. Madeo, G. Hivet, F. dell'Isola, The bias-extension test for the analysis of in-plane shear properties of textile composite reinforcements and prepregs: a review, International Journal of Material Forming 10 (4) (2017) 473-492.

[60] M. Sutton, S. McNeill, J. Helm, Y. Chao, Advances in Two-Dimensional and Three-Dimensional Computer Vision, Vol. Topics in Appl. Phys., 77, Springer, Berlin (Germany), 2000, pp. 323-372.

[61] M. Sutton, Computer vision-based, noncontacting deformation measurements in mechanics: A generational transformation, Appl. Mech. Rev. 65 (AMR-13-1009) (2013) 050802.

${ }_{695}[62]$ W. Peters, W. Ranson, Digital imaging techniques in experimental stress analysis, Opt. Eng. 21 (1982) 427-431.

[63] M. Sutton, W. Wolters, W. Peters, W. Ranson, S. McNeill, Determination of displacements using an improved digital correlation method, Im. Vis. Comp. 1 (3) (1983) 133-139. 
[64] T. Chu, W. Ranson, M. Sutton, W. Peters, Applications of digital-imagecorrelation techniques to experimental mechanics, Exp. Mech. 3 (25) (1985) 232-244.

[65] F. Hild, S. Roux, Comparison of local and global approaches to digital image correlation, Exp. Mech. 52 (9) (2012) 1503-1519.

[66] F. Hild, S. Roux, Digital image correlation, in: P. Rastogi, E. Hack (Eds.), Optical Methods for Solid Mechanics. A Full-Field Approach, Wiley-VCH, Weinheim (Germany), 2012, pp. 183-228.

[67] M. Sutton, J. Orteu, H. Schreier, Image correlation for shape, motion and deformation measurements: Basic Concepts, Theory and Applications, Springer, New York, NY (USA), 2009.

[68] H. Leclerc, J. Périé, S. Roux, F. Hild, Integrated digital image correlation for the identification of mechanical properties, in: A. Gagalowicz, W. Philips (Eds.), MIRAGE 2009, Vol. LNCS 5496, Springer, Berlin (Germany), 2009, pp. 161-171.

715 [69] J. Réthoré, S. Roux, F. Hild, An extended and integrated digital image correlation technique applied to the analysis fractured samples, Eur. J. Comput. Mech. 18 (2009) 285-306.

[70] Z. Tomičević, F. Hild, S. Roux, Mechanics-aided digital image correlation, J. Strain Analysis 48 (2013) 330-343.

[71] A. Tikhonov, V. Arsenin, Solutions of ill-posed problems, J. Wiley, New York (USA), 1977.

[72] D. Claire, F. Hild, S. Roux, A finite element formulation to identify damage fields: The equilibrium gap method, Int. J. Num. Meth. Engng. 61 (2) (2004) 189-208.

725 [73] A. Mendoza, J. Neggers, F. Hild, S. Roux, Complete Mechanical Regularization Applied to Digital Image and Volume Correlation, 
Computer Methods in Applied Mechanics and Engineering 355 (2019) 2743.

[74] M. Grédiac, F. Hild (Eds.), Full-Field Measurements and Identification in Solid Mechanics, ISTE / Wiley, London (UK), 2012.

[75] A. Carpiuc-Prisacari, M. Poncelet, K. Kazymyrenko, F. Hild, H. Leclerc, Comparison between experimental and numerical results of mixed-mode crack propagation in concrete: Influence of boundary conditions choice, Cement and Concrete Research 100 (2017) 329-340.

735

[76] F. Mathieu, H. Leclerc, F. Hild, S. Roux, Estimation of elastoplastic parameters via weighted FEMU and integrated-DIC, Exp. Mech. 55 (1) (2015) 105-119.

[77] A. Tarantola, Inverse Problems Theory. Methods for Data Fitting and Model Parameter Estimation, Elsevier Applied Science, Southampton (UK), 1987. 


\section{Appendix}

Figure 14 shows the gray level residuals for the first part of the experiment.

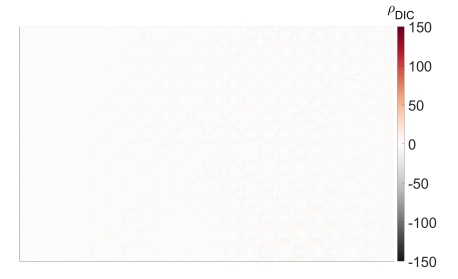

(a) DIC, \#1

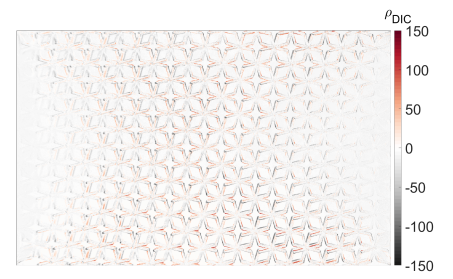

(c) DIC, \#24

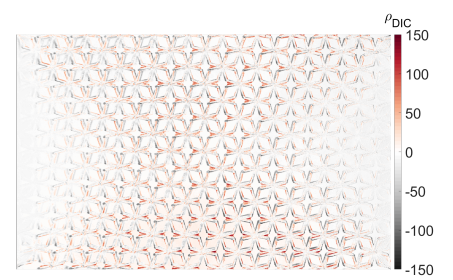

(e) DIC, \#47

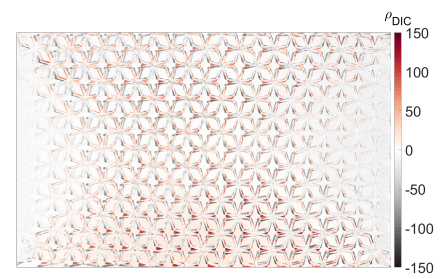

(g) DIC, \#70

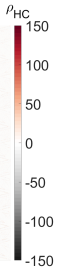

(b) $\mathrm{HC}, \# 1$

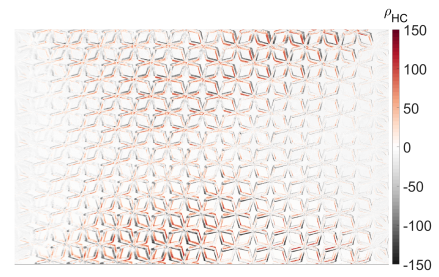

(d) $\mathrm{HC}, \# 24$

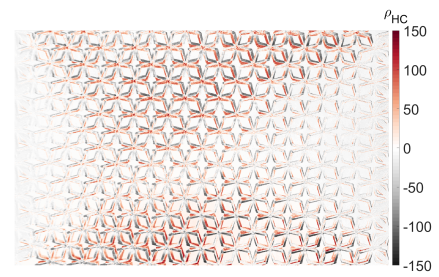

(f) $\mathrm{HC}, \# 47$

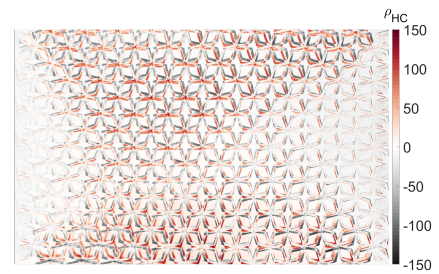

(h) $\mathrm{HC}, \# 70$

Figure 14: Gray level residual maps for direct DIC and HC predictions corresponding to pictures \#1, 24, 47 and 70 (Figure 6). 
For comparison purposes, the residual maps of the first picture are shown. They are very low and close to the acquisition noise of the camera.

Figure 15 shows the gray level residuals for the second part of the experiment. Their overall levels are higher than in the first part (Figure 14).

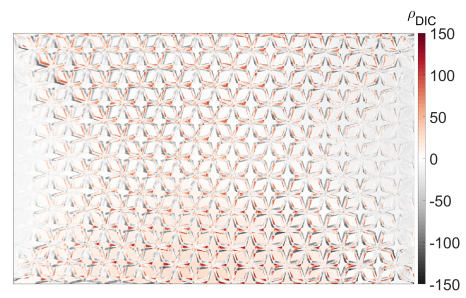

(a) DIC, \#93

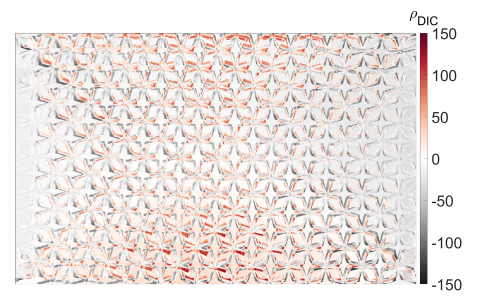

(c) DIC, \#116

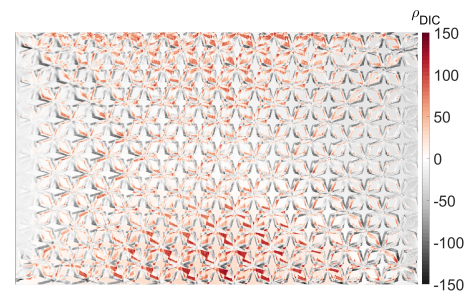

(e) DIC, \#139

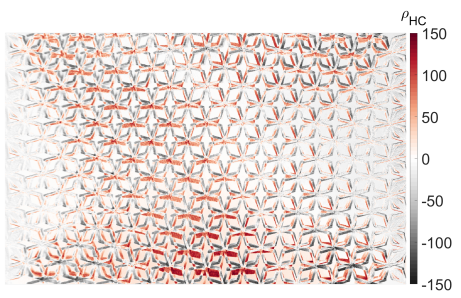

(b) $\mathrm{HC}, \# 93$

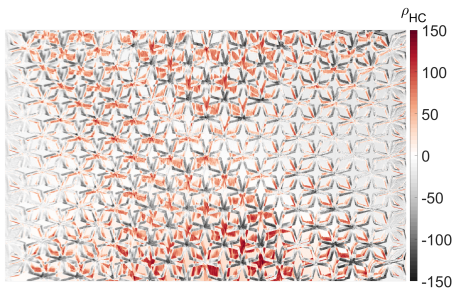

(d) HC, \#116

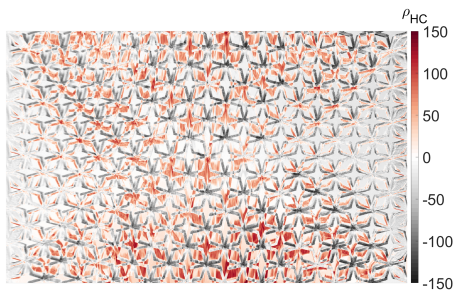

(f) $\mathrm{HC}, \# 139$

Figure 15: Gray level residual maps for direct DIC and HC predictions corresponding to pictures \#93, 116, 139 (Figure6. 